\author{
ARNAB BASU \\ TIRTHANKAR BHATTACHARYYA \\ AND \\ VIVEK S. BORKAR
}

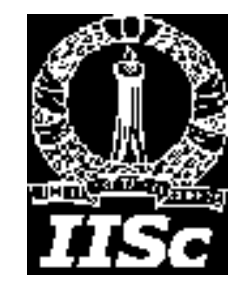

Department of Mathematics

Indian Institute of Science

Bangalore

Technical Report no. 2006/25

November 15, 2006 


\title{
A Learning Algorithm for Risk-sensitive Cost
}

\author{
Arnab Basu \\ Quantitative Methods and Information Systems Area, Indian Institute of Management Bangalore, Bannerghatta \\ Road, Bangalore 560076, India. \\ email: arnab.basu@iimb.ernet.in \\ Tirthankar Bhattacharyya \\ Department of Mathematics, Indian Insrtitute of Science, Bangalore 560012, India. \\ email: tirtha@math.iisc.ernet.in http://math.iisc.ernet.in/ tirtha \\ Vivek S. Borkar \\ School of Technology and Computer Science, Tata Institute of Fundamental Research, Homi Bhabha Road, \\ Mumbai 400005, India. \\ email: borkar@tifr.res.in http://www.tcs.tifr.res.in/ borkar
}

\begin{abstract}
A linear function approximation based reinforcement learning algorithm is proposed for Markov decision processes with infinite horizon risk-sensitive cost. Its convergence is proved using the 'o.d.e. method' for stochastic approximation. The scheme is also extended to continuous state space processes.
\end{abstract}

Key words: learning algorithm; risk-sensitive cost; function approximation; stochastic approximation

MSC2000 Subject Classification: Primary: 93E35; Secondary: 93E20, 62L20

OR/MS subject classification: Primary: Dynamic Programming / Optimal Control : Markov - Finite state and Infinite state; Secondary:

1. Introduction. Recent decades have seen a major activity in approximate dynamic programming for Markov decision processes based on real or simulated data, using reinforcement learning algorithms. (See, e.g., Bertsekas and Tsitsiklis (1996) [10] and Sutton and Barto (1998) 30 for book length treatments and $\mathrm{Si}$ et al (2004) [28 for a flavour of more recent activity. While most of this work has focused on the additive cost criteria such as discounted or time-averaged cost, relatively little has been done for the multiplicative cost (or risk-sensitive cost as it is better known). There is, however, a lot of interest in this cost criterion, as it has important applications in finance, e.g., Bagchi and Sureshkumar (2002) 2, Bielecki and Pliska (1999) [12, Bielecki and Pliska (2000) 13. In Borkar (2001) 15, Borkar (2002) [16, the 'raw' analogs of Q-learning of Watkins (1989) 32] and Actor-Critic algorithm of Barto, Sutton and Anderson (1983) [5] were developed. These were 'raw' in the sense that there was no explicit approximation of the value function in order to beat down the curse of dimensionality. In case of additive costs, there is a considerable body of work on such approximation architectures, one of the most popular being the linear function approximation. Here one seeks an approximation of the value function in terms of linear combination of a moderate number of basis functions specified a priori. The learning scheme then iteratively learns the weights (or coefficients) of this linear combination instead of learning the full value function, which is a much higher dimensional object. The first rigorous analysis of such a scheme is in Tsitsiklis and Van Roy (1997) [31, where its convergence was proved for the problem of policy evaluation. Since then there have been several variations of the basic theme, see, e.g., Bertsekas, Borkar and Nedic (2004) [ 8 and the references therein. The aim of this article is to propose a similar linear function approximation based learning scheme for policy evaluation in risk-sensitive control and justify it rigorously.

The paper is organized as follows. The next section recalls the relevant background from earlier works, specifically, Balaji and Meyn (2000) [3], Borkar (2001) [15], Borkar and Meyn (2002) [18] and Borkar (2002) [16]. Section 3 gives a motivating example from finance. Section 4 introduces the algorithm. Section 5 proves its convergence using the 'o.d.e. approach to stochastic approximation'. The latter goes back to Derevitskii and Fradkov (1974) [19] and is extensively developed in Benveniste et al (1991) 7 . A self-contained account of the relevant theory is given in the Appendix. It is an added advantage of our scheme that it easily extends to continuous state space case, which is in fact the natural setting for finance applications. Section 6 discusses this case. Section 7 concludes with some discussion.

2. Premilinaries. We begin by recalling the risk-sensitive paradigm. Let $\left\{X_{n}\right\}$ be an irreducible aperiodic Markov chain on a finite state space $S=\{1,2, \cdots, s\}$, with transition matrix $P=[[p(j \mid i)]]_{i, j \in S}$. 
While our real concern is a controlled Markov chain, we aim at a policy evaluation algorithm for a fixed stationary policy. Thus we have suppressed the explicit control dependence. The aim is to evaluate the risk-sensitive cost

$$
\lim \sup _{n \uparrow \infty} \frac{1}{n} \ln \left(E\left[e^{\sum_{m=0}^{n-1} c\left(X_{m}, X_{m+1}\right)}\right]\right) .
$$

Here $c: S \times S \rightarrow \mathcal{R}$ is a prescribed running cost function. That this limit exists follows from the multiplicative ergodic theorem for Markov chains (see Theorem 1.2 of Balaji and Meyn (2000) 3, the sufficient condition (4) therein is trivially verified for the finite state space case here). Associated with this is the multiplicative Poisson equation (see, e.g., Balaji and Meyn (2000) [3, Theorem 1.2 (ii)]):

$$
V(i)=\frac{\sum_{j} p(j \mid i) e^{c(i, j)} V(j)}{\lambda}, i \in S,
$$

with $V(\cdot), \lambda>0$. As shown in (5) of Balaji and Meyn (2000) [3], this can be explicitly given as

$$
V(i)=\lim _{n \rightarrow \infty} E\left[e^{\sum_{m=0}^{n-1}\left(c\left(X_{m}, X_{m+1}\right)-\ln (\lambda)\right)} \mid X_{0}=i\right], i \in S,
$$

where, for $\kappa_{i_{0}}=\min \left\{m>0: X_{m}=i_{0}\right\}$ with a prescribed $i_{0} \in S, \lambda$ is the unique solution to

$$
E\left[e^{\sum_{m=0}^{\kappa_{i}-1}\left(c\left(X_{m}, X_{m+1}\right)-\ln (\lambda)\right)} \mid X_{0}=i_{0}\right]=1 .
$$

(The choice of $i_{0}$ is irrelevant here.) (2) is an equation in both the vector $V=[V(1), \cdots, V(s)]$ and the scalar $\lambda$. These are respectively the Perron-Frobenius eigenvector and eigenvalue of the non-negative matrix $\left[\left[e^{c(i, j)} p(j \mid i)\right]\right]_{i, j \in S}$, whose existence is guaranteed by the Perron-Frobenius theorem. Furthermore, under our irreducibility assumption, $V$ is specified uniquely up to a positive multiplicative scalar and $\lambda$ is uniquely specified. That $\ln (\lambda)$ is in fact the cost $(1)$ then follows by Balaji and Meyn (2000) [3, Theorem $1.2]$.

The importance of (2) stems from the fact that the knowledge of $V$ is required in the policy evaluation component of the policy iteration scheme for solving the dynamic programming equation associated with risk-sensitive control (Borkar and Meyn (2002) [18, section 5]) or its 'learning' variant (Borkar (2001) [15, section 5]). One can think of an iterative 'value iteration scheme' to solve this along the lines of its nonlinear counterpart for the multiplicative dynamic programming equation for the full control problem Borkar and Meyn (2002) [18. This will be:

$$
\begin{aligned}
\tilde{V}_{n+1}(i) & =\sum_{j} p(j \mid i) e^{c(i, j)} V_{n}(j) \\
V_{n+1}(i) & =\frac{\tilde{V}_{n+1}(i)}{\tilde{V}_{n+1}\left(i_{0}\right)}
\end{aligned}
$$

where $i_{0}$ is a fixed state in $S$. This is simply a variation of the well known 'power method' for finding the dominant eigenpair of a matrix, see Stewart (2001) 29. The convergence $V_{n} \rightarrow V$ (with $V\left(i_{0}\right)=\lambda$, recall that fixing the value of $V$ at one state fixes it uniquely) and $V_{n}\left(i_{0}\right) \rightarrow \lambda$ can be proved by standard linear algebra arguments, but also follows as a special case of Borkar and Meyn (2002) [18, Theorem 4.5] which handles a nonlinear (and therefore more complicated) version arising from dynamic programming.

It should be noted that the 'normalization' by $\tilde{V}_{n+1}\left(i_{0}\right)$ above is just one among many possible alternatives. The algorithm seeks a ray $V^{*}$ in the positive cone, which can be identified by any nonzero point on it. The above scheme will identify the ray by specifying the point on it corresponding to $V^{*}\left(i_{0}\right)=\lambda$. Another alternative could have been, e.g., to normalize by $\sum_{i} \tilde{V}_{n+1}(i)$, which would have identified the point corresponding to $\sum_{i} V^{*}(i)=\lambda$. This situation is completely analogous to a similar situation arising in the relative value iteration for ergodic control, of which this is the multiplicative analog.

Following Borkar (2001) [15] and Borkar (2002) [16], one can formulate a 'learning' version of this by writing down its stochastic approximation counterpart:

$$
V_{n+1}(i)=V_{n}(i)+a(n)\left(\frac{e^{c\left(i, Z_{n+1}^{i}\right)} V_{n}\left(Z_{n+1}^{i}\right)}{V_{n}\left(i_{0}\right)}-V_{n}(i)\right),
$$

with $a(n)>0 \forall n, \sum_{n} a(n)=\infty, \sum_{n} a(n)^{2}<\infty$, and $Z_{n+1}^{i}$ is a random variable generated according to the law $p(\cdot \mid i)$ by a simulation device, independent of all else. This is the 'synchronous' version as 
in Borkar (2002) [16, wherein all components are updated concurrently. It requires the generation of $s$ random variables $Z_{n}^{i}, i \in S$, at the $n$-th iterate. The 'asynchronous' version based on a single simulation run of the Markov chain $\left\{X_{n}\right\}$ would update only the $X_{n}$-th component at time $n$. It is given by:

$$
V_{n+1}(i)=V_{n}(i)+a(n) I\left\{X_{n}=i\right\}\left(\frac{e^{c\left(X_{n}, X_{n+1}\right)} V_{n}\left(X_{n+1}\right)}{V_{n}\left(i_{0}\right)}-V_{n}(i)\right) .
$$

This can be considered as an uncontrolled version of the Q-learning algorithm proposed and analyzed in Borkar (2002) [16 by taking the action space to be a singleton. Note that we have used the normalization by $V_{n}\left(i_{0}\right)$ rather than an appropriately defined $\tilde{V}_{n+1}\left(i_{0}\right)$, in order to be faithful to Borkar (2001) [15], Borkar (2002) [16. The difference between the two is asymptotically negligible due to the diminishing stepsize, whereas the present formulation is more convenient for the iterative scheme.

Consider the synchronous case. To mimic the proof technique of Borkar (2002) [16, one would first argue that this iteration tracks with probability one the asymptotic behavior of the associated ('primary') o.d.e. limit

$$
\dot{V}_{i}(t)=\frac{\sum_{j} p(j \mid i) e^{c(i, j)} V_{j}(t)}{V_{i_{0}}(t)}-V_{i}(t), i \in S .
$$

There is another associated o.d.e., termed the 'secondary o.d.e.' in Borkar (2002) [16], given by

$$
\dot{V}_{i}(t)=\frac{\sum_{j} p(j \mid i) e^{c(i, j)} V_{j}(t)}{\lambda}-V_{i}(t), i \in S .
$$

The secondary o.d.e. presupposes the knowledge of $\lambda$ and therefore cannot be used for algorithmic purposes. The proof of Borkar (2002) [16] works by first establishing the convergence of the secondary o.d.e. and then inferring that of the primary o.d.e. by exploiting certain relationships between the two. We follow a similar strategy here to analyze the linear function approximation based scheme proposed next.

3. An example. Let an investor's portfolio consist only of $d$ stocks and one money market account. The stock prices follow a $d$-dimensional "geometric Brownian motion" $s(t) \stackrel{\text { def }}{=}\left(s^{(1)}(t), \ldots, s^{(d)}(t)\right)^{T}$ given by the following stochastic differential equation :

$$
(\operatorname{diag}(s(t)))^{-1} d s(t)=\tilde{b} d t+\tilde{\sigma} d \tilde{W}(t)
$$

and the money market account $s^{(0)}(t)$ follows the equation :

$$
d s^{(0)}(t)=\bar{r} s^{(0)}(t) d t
$$

where $\tilde{W}(\cdot) \stackrel{\text { def }}{=}\left[\tilde{W}_{1}(\cdot), \ldots, \tilde{W}_{d}(\cdot)\right]^{T}$ is a $d$-dimensional standard Brownian Motion and the interest rate $\bar{r} \geq 0$, the drift vector $\tilde{b} \stackrel{\text { def }}{=}\left[\tilde{b}_{1}, \ldots, \tilde{b}_{d}\right]^{T} \in \mathcal{R}^{d}$ and the diffusion matrix

$$
\tilde{\sigma} \stackrel{\text { def }}{=}\left[\begin{array}{ccc}
\tilde{\sigma}_{11} & \ldots & \tilde{\sigma}_{1 d} \\
\vdots & \vdots & \vdots \\
\tilde{\sigma}_{d 1} & \ldots & \tilde{\sigma}_{d d}
\end{array}\right] \in \mathcal{R}^{d \times d},
$$

are assumed to be known.

The investor is trying to optimize his 'returns' under a 'safe policy' of always keeping some money in his money market account and distributing the rest over all assets in an optimal way. Specifically, he first ensures that a fixed small amount $\delta>0$ is taken from the total wealth (denoted by $V(\cdot)$ ) and put into the money market account, and then manipulates the fraction of his remaining total wealth in any particular asset $i$, given by $u^{(i)}(\cdot) \stackrel{\text { def }}{=} \frac{N_{i}(\cdot) s^{(i)}(\cdot)}{V(\cdot)-\delta}$. Here $N_{i}(t) \stackrel{\text { def }}{=}$ number of units of asset $i$ held at time $t$. Thus we can assume that the investor's control process $u(\cdot)$ takes values in $U \stackrel{\text { def }}{=}\left\{\left(u^{(0)}, u^{(1)}, \ldots, u^{(d)}\right) \in\right.$ $\left.[0,1]^{d+1}: \sum_{i=0}^{d} u^{(i)}=1\right\}$. By definition, $U$ is compact.

Under these assumptions, the "remaining" wealth process of the investor, defined as $\hat{V}(t) \stackrel{\text { def }}{=}$ $\sum_{i=0}^{d} N_{i}(t) s^{(i)}(t) \equiv V(t)-\delta$, follows the s.d.e.:

$$
\frac{d \hat{V}(t)}{\hat{V}(t)}=\langle u(t),(\bar{r}, \tilde{b})\rangle d t+\left\langle\tilde{\sigma}^{\prime} u^{[1: d]}(t), d \tilde{W}(t)\right\rangle .
$$


Thus the total wealth process $V(\cdot)$ follows the s.d.e.:

$$
\frac{d V(t)}{V(t)-\delta}=\langle u(t),(\bar{r}, \tilde{b})\rangle d t+\left\langle\tilde{\sigma}^{\prime} u^{[1: d]}(t), d \tilde{W}(t)\right\rangle .
$$

By a standard argument using the martingale representation theorem, we write the above equation for $V(t)$ as follows :

$$
d V(t)=b(V(t), u(t)) d t+\sigma(V(t), u(t)) d W(t)
$$

where $b(\cdot): \mathcal{R} \times U \rightarrow \mathcal{R}, \sigma(\cdot): \mathcal{R} \times U \rightarrow \mathcal{R}$ are given by

$$
\begin{array}{ccc}
b(V(t), u(t)) \stackrel{\text { def }}{=} & (V(t)-\delta)\langle u(t),(\bar{r}, \tilde{b})\rangle, \\
\sigma(V(t), u(t)) \stackrel{\text { def }}{=} & (V(t)-\delta)\left\|\tilde{\sigma}^{\prime} u^{[1: d]}(t)\right\|
\end{array}
$$

and $W(\cdot)$ is a standard 1-dimensional Brownian motion on a possibly enlarged probability space. Note that starting with an initial wealth $V(0)=x>\delta$, it follows from equation (6) above that $V(\cdot) \geq \delta$ a.s. for any control process $u(\cdot)$. (see, e.g., Karatzas and Shreve (1988) [24, Chapter 5]).

We truncate and discretize the state space as $S_{h} \stackrel{\text { def }}{=}\{\delta, \delta+h, \delta+2 h, \ldots, \delta+N h\}$. Given a fixed stationary control process $u(\cdot)$ as per the above formulation and the corresponding portfolio process 6 . with initial value $V(0)=x>0$, we can exactly mimic the arguments of Examples 3 and 4 of Kushner and Dupuis (2001) [27, pp. 95-98] to get an approximating Markov chain $\left\{V_{n}^{h}\right\}_{n=0,1, \ldots}$ on the finite state space $S_{h}$ which is locally consistent with [6] in the sense of Kushner and Dupuis (2001) [27].

Our aim now is to estimate the corresponding risk-sensitized expected exponential portfolio growth rate, also called the volatility adjusted geometric mean return for the above portfolio $V(\cdot)$, defined as:

$$
J_{\theta}(x) \stackrel{\text { def }}{=} \liminf _{t \uparrow \infty} \frac{-2}{\theta} \frac{1}{t} \ln E\left[e^{-\frac{\theta}{2} \ln V(t)} \mid V(0)=x\right]
$$

where $\theta \in(0, \infty)$ can be interpreted as the risk-sensitivity parameter or risk-aversion parameter, for the bigger its value the bigger the penalty associated with the portfolio's risk. For economic justification of the risk-sensitive cost, refer to Bielecki and Pliska (2003) [14].

Given the above locally consistent approximating Markov chain $\left\{V_{n}^{h}\right\}$, our aim thus reduces to estimating the corresponding analogue of (7) given by:

$$
J_{\theta}^{h}(x) \stackrel{\text { def }}{=} \liminf _{n \uparrow \infty} \frac{-2}{\theta} \frac{1}{n} \ln E\left[e^{-\frac{\theta}{2} \ln V_{n}^{h}} \mid V_{0}^{h}=x\right]
$$

Writing $\ln V_{n}^{h}$ as a telescoping series $\sum_{m=0}^{n-1} \ln \left(\frac{V_{m+1}^{h}}{V_{m}^{h}}\right)$ puts this in the form 1 under consideration.

4. The algorithm. The above scheme, as mentioned earlier, suffers from the curse of dimensionality. This motivates the linear function approximation

$$
V(i) \approx \sum_{k=1}^{M} r^{k} \phi_{k}(i)=\phi^{T}(i) r
$$

where $r=\left[r^{1}, \cdots, r^{M}\right]^{T}$ is a vector of coefficients and $\phi^{k}(\cdot), 1 \leq k \leq M$, are basis functions, or features, chosen a priori. Set $\phi(i)=\left[\phi^{1}(i), \cdots, \phi^{M}(i)\right]^{T}$. Here $M$ is typically significantly smaller than $s$. Define the $s \times M$ matrix $\Phi$ whose $(i, k)$-th entry is $\phi^{k}(i)$ for $1 \leq i \leq s$ and $1 \leq k \leq M$.

We make the following important assumption:

$(\dagger) \phi^{1}, \cdots, \phi^{M}$ are orthogonal vectors in the positive cone of $\mathcal{R}^{s}$, and the submatrix of $P$ corresponding to $\cup_{k}\left\{i: \phi^{k}(i)>0\right\}$ is irreducible.

Under this, $\Phi$ has the full rank $M$.

REMARK 4.1 Note that $(\dagger)$ is a stronger condition than what is used in the additive cost cases mentioned earlier, where only linear independence is required. It will be seen to play a key role in our analysis (see the proof of Lemma 5.1 below), where it ensures that the learning remains within the positive cone. 
REMARK 4.2 Let $e_{i}, 1 \leq i \leq s$, denote the unit vectors in the coordinate directions and $\phi^{k}=$ $\sum_{j=1}^{s} a_{k j} e_{j}, 1 \leq k \leq M$. Then clearly $a_{k j} \geq 0 \forall k, j$. However, (†) perforce implies that for $i \neq k$, the sets $\left\{j: a_{i j}>0\right\}$ and $\left\{j: a_{k j}>0\right\}$ are disjoint. That is, the $\phi^{k}$ 's can be viewed as a weighted aggregation of states, though their original (problem specific) interpretation may be different.

The algorithm we propose is an analog of the 0-LSPE scheme in Bertsekas and Nedic (2003) [9] and Bertsekas, Borkar and Nedic (2004) 8]. It is given by

$$
r_{n+1}=r_{n}+a(n)\left(\frac{B_{n}^{-1} A_{n}}{\phi^{T}\left(i_{0}\right) r_{n}}-I\right) r_{n}, n \geq 0,
$$

where

$$
A_{n} \stackrel{\text { def }}{=} \sum_{m=0}^{n} e^{c\left(X_{m}, X_{m+1}\right)} \phi\left(X_{m}\right) \phi^{T}\left(X_{m+1}\right), \quad B_{n} \stackrel{\text { def }}{=} \sum_{m=0}^{n} \phi\left(X_{m}\right) \phi^{T}\left(X_{m}\right) .
$$

Also, $i_{0}$ is a fixed state in $\cup_{k}\left\{j: \phi^{k}(j)>0\right\}$. Note that both $\left\{A_{n}\right\}$ and $\left\{B_{n}^{-1}\right\}$ can be computed iteratively by

$$
\begin{aligned}
& A_{n+1}=A_{n}+e^{c\left(X_{n}, X_{n+1}\right)} \phi\left(X_{n+1}\right) \phi^{T}\left(X_{n+2}\right), \\
& B_{n+1}^{-1}=B_{n}^{-1}-\frac{B_{n}^{-1} \phi\left(s_{n+1}\right) \phi^{T}\left(s_{n+1}\right) B_{n}^{-1}}{1+\phi^{T}\left(X_{n+1}\right) B_{n}^{-1} \phi\left(X_{n+1}\right)},
\end{aligned}
$$

where the latter follows from the Sherman-Morrison-Woodbury formula (Golub and Van Loan (1996), [22, Page 3]). If $B_{n}$ is not invertible in early iterations, one may add to it $\delta$ times the identity matrix for a small $\delta>0$, or use pseudo-inverse in place of inverse. Such modifications are inconsequential in the long run due to time averaging implicit in the scheme, as will become apparent later (see (12) below). See also Bertsekas and Nedic (2003) [9], Bertsekas, Borkar and Nedic (2004) 8] for similar situations arising in the additive cost context. Note also that the matrices being inverted in the above iteration are diagonal and therefore their inversion is computationally easy.

To avoid 'division by zero', we modify (9) as

$$
r_{n+1}=r_{n}+a(n)\left(\frac{B_{n}^{-1} A_{n}}{\left(\phi^{T}\left(i_{0}\right) r_{n}\right) \vee \epsilon}-I\right) r_{n}, n \geq 0,
$$

where $\epsilon>0$ is a small prescribed scalar.

Define the $s \times s$ matrices

$$
D \stackrel{\operatorname{def}}{=}\left[\begin{array}{cccc}
\pi(1) & 0 & \cdots & 0 \\
0 & \pi(2) & \cdots & 0 \\
0 & 0 & \cdots & 0 \\
0 & 0 & \cdots & \pi(s)
\end{array}\right], C \stackrel{\operatorname{def}}{=}\left[\begin{array}{cccc}
e^{c(1,1)} & e^{c(1,2)} & \cdots & e^{c(1, s)} \\
e^{c(2,1)} & e^{c(2,2)} & \cdots & e^{c(2, s)} \\
\cdots & \cdots & \cdots & \cdots \\
e^{c(s, 1)} & e^{c(s, 2)} & \cdots & e^{c(s, s)}
\end{array}\right],
$$

where $\pi=[\pi(1), \cdots, \pi(s)]$ denotes the unique stationary distribution of $\left\{X_{n}\right\}$. Let $\|\cdot\|_{D}$ denote the norm induced by the inner product $\langle x, y\rangle \stackrel{\text { def }}{=} x^{T} D y$ and $L_{2}(S, D)$ the corresponding Hilbert space. Let $X \stackrel{\text { def }}{=}\left\{\sqrt{D} \Phi r: r \in \mathcal{R}^{M}\right\}$ and $\Pi$ the projection from $\mathcal{R}^{s}$ onto $X$ w.r.t. the above norm, given by

$$
\Pi=\sqrt{D} \Phi\left(\Phi^{T} D \Phi\right)^{-1} \Phi^{T} \sqrt{D} .
$$

Next, define

Then 10 may be rewritten as

$$
\bar{B}_{n} \stackrel{\text { def }}{=} \frac{B_{n}}{n+1}, \quad \bar{A}_{n} \stackrel{\text { def }}{=} \frac{A_{n}}{n+1} .
$$

$$
r_{n+1}=r_{n}+a(n)\left(\frac{\bar{B}_{n}^{-1} \bar{A}_{n}}{\left(\phi^{T}\left(i_{0}\right) r_{n}\right) \vee \epsilon}-I\right) r_{n}, n \geq 0 .
$$

By the ergodicity of $\left\{X_{n}\right\}$, we have

$$
\bar{B}_{n} \rightarrow B, \quad \bar{A}_{n} \rightarrow A \text { a.s. }
$$

where

$$
B \stackrel{\text { def }}{=} \Phi^{T} D \Phi, \quad A \stackrel{\text { def }}{=} \Phi^{T} D C \circ P \Phi,
$$


and ' $\circ$ ' denotes the componentwise product of two matrices with identical row and column dimensions. Then (11) may be further rewritten as

$$
r_{n+1}=r_{n}+a(n)\left(\frac{B^{-1} A}{\left(\phi^{T}\left(i_{0}\right) r_{n}\right) \vee \epsilon}-I\right) r_{n}+a(n) Z_{n} r_{n}, n \geq 0
$$

where

$$
Z_{n} \stackrel{\text { def }}{=} \frac{\bar{B}_{n}^{-1} \bar{A}_{n}-B^{-1} A}{\phi^{T}\left(i_{0}\right) r_{n} \vee \epsilon} \rightarrow 0, \quad \text { a.s. }
$$

by virtue of 12 .

We conclude this section with some comments on the approximation error. The quantity of interest here will turn out to be $\left|\gamma^{*}-\hat{\gamma}\right|$, where $\hat{\gamma}$ is the Perron-Frobenius eigenvalue of $C \circ P$, equivalently, of $\mathcal{M}=\sqrt{D} C \circ P \sqrt{D}^{-1}$, and $\gamma^{*}$ is that of $\Pi \mathcal{M}$. As an immediate consequence of Bhatia (1997) [11, Theorem VII.1.1, p. 227], we have

$$
\left|\gamma^{*}-\hat{\gamma}\right| \leq(\|C \circ P\|+\|\Pi \mathcal{M}\|)^{1-\frac{1}{s}}\|C \circ P-\Pi \mathcal{M}\|^{\frac{1}{s}} .
$$

$\|$. $\|$ here denotes the usual matrix norm. This, however, is not a very strong bound, particularly for large $s$ which is the case of interest here. Some additional insight can be gained by considering the special case when $C \circ P$ is symmetric. Let $\gamma_{1}>\gamma_{2},>\cdots>\gamma_{s}$ denote its eigenvalues. Then the best scenario is when we pick the Perron-Frobenius eigenvector as one of the features, whence the error is in fact zero ! The worst, of course, is the situation when we pick the $M$ features to be the eigenvectors corresponding to the $M$ smallest eigenvectors, in which case it is $\hat{\gamma}-\gamma_{s-M+1}$. Such an extreme case does not occur in our set-up because the Perron-Frobenius vector makes an acute angle with the positive cone formed by the features. Nevertheless, this clearly brings out the critical role of feature selection.

It may be noted also that, since any irreducible nonnegative aperiodic matrix can be written as a product of a diagonal matrix with positive diagonal entries and an irreducible stochastic aperiodic matrix, the above is in fact a general scheme for estimating the Perron-Frobenius eigenvalue of a general irreducible and aperiodic nonnegative matrix. This has applications in domains other than control as well.

5. Convergence analysis We shall prove convergence of the above scheme assuming for the time being the 'stability of iterates':

$$
\sup _{n}\left\|r_{n}\right\|<\infty, \quad \text { a.s. }
$$

We prove this later (Lemma 5.5 below). Under (14), the iteration (13) has the same asymptotic behavior as the o.d.e. (see Lemma A.1 of the Appendix)

$$
\dot{r}(t)=\left(\frac{B^{-1} A}{\phi^{T}\left(i_{0}\right) r(t) \vee \epsilon}-I\right) r(t) .
$$

We begin by analyzing the $\epsilon=0$ case, i.e.,

$$
\dot{r}(t)=\left(\frac{B^{-1} A}{\phi^{T}\left(i_{0}\right) r(t)}-I\right) r(t) .
$$

Let $W \stackrel{\text { def }}{=} \sqrt{D} \Phi$ and $\mathcal{M} \stackrel{\text { def }}{=} \sqrt{D} C \circ P \sqrt{D}^{-1}$. Then $B=W^{T} W$ and $\Pi=W\left(W^{T} W\right)^{-1} W^{T}$. Setting $Y(t) \stackrel{\text { def }}{=} W r(t), 16$ may be rewritten as

$$
\dot{Y}(t)=\left(\frac{\Pi \mathcal{M}}{\phi^{T}\left(i_{0}\right) r(t)}-I\right) Y(t)=\left(\sqrt{\pi\left(i_{0}\right)} \frac{\Pi \mathcal{M}}{Y_{i_{0}}(t)}-I\right) Y(t) .
$$

Note that $\mathcal{M}$ is a nonnegative matrix. It is worth noting here that for $s=M$ and $D=$ the identity matrix, 17) reduces to 3 as it should. The occurence of $D$ is due to the asynchronous implementation: the state $i$ gets sampled, and therefore the $i$-th component gets updated with relative frequency $=$ the stationary distribution of $i$.

Let $\mathcal{P}$ denote the positive cone in $X$ defined by $\stackrel{\text { def }}{=} X \cap \mathcal{R}_{+}^{s}$. 
LeMma 5.1 For $Q \stackrel{\text { def }}{=} \Pi \mathcal{M}$,

(i) $Q$ maps $\mathcal{R}_{+}^{s}$ into $\mathcal{P}$ and

(ii) $Q$ has a Perron-Frobenius eigenvalue $\gamma^{*}>0$ with an associated nonnegative eigenvector $Y^{*}$ such that $Y^{*}(i)>0$ for $i \in \cup_{k}\left\{j: \phi^{k}(j)>0\right\}$.

Proof. $\mathcal{M}$ is a nonnegative matrix. Under our choice of the $\phi^{i}$ s,,$\left(W^{T} W\right)^{-1}=\left(\Phi^{T} D \Phi\right)^{-1}$ is diagonal with positive entries on the diagonal. Thus $\Pi$ and therefore $Q$ is a nonnegative matrix. The first claim follows. By the latter part of $(\dagger)$, it follows that the submatrix of $Q$ corresponding to $i \in \cup_{k}\left\{j: \phi^{k}(j)>0\right\}$ is irreducible and the remaining rows and columns are zero. The second claim now follows from the Perron-Frobenius theorem.

Consider the secondary o.d.e.

$$
\dot{Y}(t)=\left(\frac{Q}{\gamma^{*}}-I\right) Y(t)
$$

We shall analyze 18 under the condition

$$
Y(0)=\sqrt{D} \Phi r(0) \in \operatorname{int}(\mathcal{P}), \quad \phi^{T}\left(i_{0}\right) r(0)>0,
$$

where $\operatorname{int}(\mathcal{P})$ denotes the interior of $\mathcal{P}$. Let

$$
H \stackrel{\text { def }}{=}\left\{Y: Q Y=\gamma^{*} Y, Y \geq 0 \text { (componentwise), } Y\left(i_{0}\right)>0\right\} .
$$

Lemma 5.2 Every solution of (18) initiated in the positive cone converges to $H$.

Proof. Since $\gamma^{*}$ is the Perron-Frobenius eigenvalue of $Q$, the matrix $\left(\left(\gamma^{*}\right)^{-1} Q-I\right)$ has zero as one eigenvalue and the rest have negative real parts. By standard linear system theory, the trajectory will converge exponentially to the one dimensional subspace spanned by the eigenvector of $\left(\left(\gamma^{*}\right)^{-1} Q-I\right)$ corresponding to the zero eigenvalue, i. e., the right Perron-Frobenius eigenvector $\tilde{Y}$ of $Q$. We show that a solution initiated in the positive cone $\mathcal{P}$ does not leave $\mathcal{P}$. Suppose it does. Let $\tau=\inf \{t>0: Y(t) \notin \mathcal{P}\}$ $\left(=\infty\right.$ if this set is empty). If $\tau<\infty$, then at $t=\tau, Y_{i}(t)=0$ for some $i$. But then the $i$-th component of the r.h.s. of (18) is non-negative for all such $i$ (again, by Lemma 5.1) and therefore none of these components can go negative immediately after $t$. This implies that $\tau=\infty$. Ler $y$ be the left PerronFrobenius eigenvector of $Q$. Then $y(i)>0$ for $i \in \cup_{k}\left\{j: \phi^{k}(j)>0\right\}$. From (18), we have $y^{T} Y(t)$ is a constant, in particular, $y^{T} Y(t)=y^{T} Y(0) \geq \sqrt{\pi\left(i_{0}\right)} \phi^{T}\left(i_{0}\right) r(0)>0$. Thus the limit $\hat{Y}$ of $Y(t)$ as $t \uparrow \infty$ must also satisfy $y^{T} \hat{Y}>0$. In particular, it is a nonzero multiple of $\tilde{Y}$, for which the $i$-th component for $i \in \cup_{k}\left\{j: \phi^{k}(j)>0\right\}$ will be strictly positive. In particular, this is so for $i=i_{0}$. The claim follows.

We shall now analyze (17) under the condition

$$
\sqrt{D} \Phi r(0) \in \operatorname{int}(\mathcal{P}), \quad \phi^{T}\left(i_{0}\right) r(0)>0 .
$$

Again, let $\tau=\inf \{t>0: Y(t) \notin \mathcal{P}\}(=\infty$ if this set is empty).

Lemma $5.3 \phi^{T}\left(i_{0}\right) r(t)>0$ and $\sqrt{D} \Phi r(t) \in \mathcal{P}$ for all $t>0$.

Proof. We first eliminate the possibility that $\lim _{t \uparrow T}\|Y(t)\|=\infty$ for some finite $T$. For this, it suffices to show that $\phi^{T}\left(i_{0}\right) r(t)$ remains bounded away from zero on any finite time interval, because then a standard argument using the Gronwall inequality will imply that the entire trajectory is bounded on this interval. Thus if there did exist some such $T<\infty$, then for some $T^{\prime}<\infty, \lim _{t \uparrow T^{\prime}} \phi^{T}\left(i_{0}\right) r(t)=0$. But on $\left[0, T^{\prime}\right)$,

$$
\dot{Y}_{i_{0}}(t) \geq-Y_{i_{0}}(t)
$$

in view of Lemma 5.1, and hence

$$
Y_{i_{0}}(t)=\sqrt{\pi\left(i_{0}\right)} \phi^{T}\left(i_{0}\right) r(t) \geq e^{-t} Y_{i_{0}}(0)=e^{-t} \sqrt{\pi\left(i_{0}\right)} \phi^{T}\left(i_{0}\right) r(0),
$$

which contradicts the condition that $\lim _{t \uparrow T^{\prime}} \phi^{T}\left(i_{0}\right) r(t)=0$. Thus there is no such $T^{\prime}$ and $\phi^{T}\left(i_{0}\right) r(t)>0$ for all $t>0$. As in the preceding lemma, if $\tau<\infty$, then at $t=\tau, Y_{i}(t)=0$ for some $i$. But then the $i$-th component of the r.h.s. of (17) is non-negative for all such $i$ (again, by Lemma 5.1) and therefore 
none of these components can go negative immediately after $t$. This implies that $\tau=\infty$ and the second claim follows.

The proof of the next lemma mimics the arguments of Borkar (2002) [16].

Lemma 5.4 Trajectories of (17) remain bounded and converge to a Perron-Frobenius eigenvector of $Q$.

Proof. Let $Y(\cdot)$ be a trajectory of (17) satisfying (20). Set

$$
\nu(t) \stackrel{\text { def }}{=} \frac{\phi^{T}\left(i_{0}\right) r(t)}{\gamma^{*}}, \tau(t) \stackrel{\text { def }}{=} \int_{0}^{t} \frac{1}{\nu(s)} d s, \quad t \geq 0 .
$$

As $\nu(t)>0$ for $t \geq 0$, this is well-defined. The map $t \rightarrow \tau(t)$ is monotone strictly increasing, and thus has an inverse from its range to $[0, \infty)$. Let $\rho(t) \stackrel{\text { def }}{=} \nu\left(\tau^{-1}(t)\right)$, i.e., $\rho(\tau(t))=\nu(t)$. Let $\tilde{Y}(\cdot)$ be a trajectory of 18 with $\tilde{Y}(0)=Y(0)$ and set $\bar{Y}(t)=\exp \left(\int_{0}^{t}(1-\rho(s)) d s\right) \tilde{Y}(t), t \geq 0$. Then $\bar{Y}(0)=Y(0)$. Explicit calculations show that

$$
\dot{\bar{Y}}(t)=\rho(t)\left(\frac{1}{\rho(t)} \frac{Q \bar{Y}(t)}{\gamma^{*}}-\bar{Y}(t)\right) .
$$

$Y^{\prime}(\cdot) \stackrel{\text { def }}{=} \bar{Y}(\tau(\cdot))$ satisfies

$$
\begin{aligned}
\dot{Y}^{\prime}(t) & =\dot{\tau}(t) \rho(\tau(t))\left(\frac{1}{\rho(\tau(t))} \frac{Q Y^{\prime}(t)}{\gamma^{*}}-Y^{\prime}(t)\right) \\
& =\frac{1}{\nu(t)} \frac{Q Y^{\prime}(t)}{\gamma^{*}}-Y^{\prime}(t)
\end{aligned}
$$

which is the same as 17]. By standard uniqueness result for o.d.e.s, $Y(\cdot)=Y^{\prime}(\cdot)=\psi(\cdot) \tilde{Y}(\tau(\cdot))$, where $\psi(t) \stackrel{\text { def }}{=} \exp \left(\int_{0}^{\tau(t)}(1-\rho(s)) d s\right), t \geq 0$. Thus

$$
\frac{\sqrt{\pi\left(i_{0}\right)} Q Y(t)}{Y_{i_{0}}(t)}=\frac{\sqrt{\pi\left(i_{0}\right)} Q \tilde{Y}(\tau(t))}{\tilde{Y}_{i_{0}}(\tau(t))} .
$$

By Lemma 5.2, this remains bounded for all $t>0$. Since 17 implies that

$$
Y(t)=Y(0)+\int_{0}^{t} e^{-(t-s)} \frac{\sqrt{\pi\left(i_{0}\right)} Q Y(s)}{Y_{i_{0}}(s)} d s,
$$

it follows that $Y(t)$ and therefore $\nu(t)$ remains bounded for all $t \geq 0$. In particular, $\tau(t) \geq \beta t$ for some $\beta>0$ and thus $\tau(t) \uparrow \infty$ as $t \uparrow \infty$. By Lemma $5.2, \tilde{Y}(t) \rightarrow$ some $\bar{Y}^{*} \in H$. Thus

$$
\lim _{t \rightarrow \infty} \frac{\sqrt{\pi\left(i_{o}\right)} Q Y(t)}{Y_{i_{0}}(t)}=\lim _{t \rightarrow \infty} \frac{\sqrt{\pi\left(i_{o}\right)} Q \tilde{Y}(t)}{\tilde{Y}_{i_{0}}(t)}=\frac{\sqrt{\pi\left(i_{o}\right)} Q Y^{*}}{Y_{i_{0}}^{*}} .
$$

Thus any limit of $Y(t+\cdot)$ in $C\left([0, \infty) ; \mathcal{R}^{s}\right)$ as $t \uparrow \infty$ must be a trajectory of

$$
\dot{y}(t)=\frac{\sqrt{\pi\left(i_{o}\right)} Q Y^{*}}{Y_{i_{0}}^{*}}-y(t)=\frac{\sqrt{\pi\left(i_{0}\right)} \gamma^{*} Y^{*}}{Y_{i_{0}}^{*}}-y(t),
$$

lying in an invariant set thereof. But this o.d.e. has only one invariant set 1 , viz., the singleton $\left\{\frac{\sqrt{\pi\left(i_{0}\right)} \gamma^{*} Y^{*}}{Y_{i_{0}}^{*}}\right\}$. Therefore $Y(t)$ converges to this point as $t \uparrow \infty$. In particular, $Y_{i_{0}}(t)$ converges to $\sqrt{\pi\left(i_{0}\right)} \gamma^{*}$, implying that $Y(t)$ converges to $c Y^{*}$ where $c$ is such that $Y_{i_{0}}^{*}=\sqrt{\pi\left(i_{0}\right)} \gamma^{*}$.

Corollary 5.1 For $r(\cdot)$ as in 16$), \phi^{T}\left(i_{0}\right) r(t) \rightarrow \gamma^{*}$ as $t \uparrow \infty$.

Let $G=\left\{x \in H: x\left(i_{0}\right)=\sqrt{\pi\left(i_{0}\right)} \gamma^{*}\right\}$

THEOREM $5.1 G$ is the globally exponentially stable attractor for (17).

\footnotetext{
${ }^{1}$ other than the whole space
} 
Proof. From Lemma 5.4, trajectories of (17) converge to $G$. In the notation of Lemma 5.4,

$$
\frac{\sqrt{\pi\left(i_{o}\right)} Q Y(t)}{Y_{i_{0}}(t)}=\frac{\sqrt{\pi\left(i_{o}\right)} Q \tilde{Y}(\tau(t))}{\tilde{Y}_{i_{0}}(\tau(t))} .
$$

Thus

$$
\begin{aligned}
Y(t)= & e^{-t} Y(0)+\int_{0}^{t} e^{-(t-s)} \frac{\sqrt{\pi\left(i_{o}\right)} Q \tilde{Y}(\tau(s))}{\tilde{Y}_{i_{0}}(\tau(s))} d s \\
= & e^{-t} Y(0)+\int_{0}^{t} e^{-(t-s)}\left(\frac{\sqrt{\pi\left(i_{o}\right)} Q \tilde{Y}(\tau(s))}{\tilde{Y}_{i_{0}}(\tau(s))}-\frac{\sqrt{\pi\left(i_{o}\right)} Q Y^{*}}{Y_{i_{0}}^{*}}\right) d s \\
& +\int_{0}^{t} e^{-(t-s)} \frac{\sqrt{\pi\left(i_{o}\right)} Q Y^{*}}{Y_{i_{0}}^{*}} d s .
\end{aligned}
$$

Since the convergence $\tilde{Y}(t) \rightarrow Y^{*}$ is exponential and $\tau(t) \geq \beta t$ for some $\beta>0$, it follows that the term in parentheses in the first integrand tends to zero exponentially as $s \uparrow \infty$. Thus the first integral on the right tends to zero exponentially. Now

$$
\frac{\sqrt{\pi\left(i_{0}\right)} Q Y^{*}}{Y_{i_{0}}^{*}}=\frac{\sqrt{\pi\left(i_{0}\right)} \gamma^{*} Y^{*}}{Y_{i_{0}}^{*}} \in G
$$

Thus $Y(t) \rightarrow G$ exponentially.

Going back to 15 , let $r^{\epsilon}(\cdot)$ denote its solution and let $Y^{\epsilon}=W r^{\epsilon}(\cdot)$. Then

$$
\dot{Y}^{\epsilon}(t)=\left(\frac{\sqrt{\pi\left(i_{0}\right)} \Pi \mathcal{M}}{Y_{i_{0}}^{\epsilon}(t) \vee \epsilon^{\prime}}-I\right) Y^{\epsilon}(t),
$$

where $\epsilon^{\prime}=\sqrt{\pi\left(i_{0}\right)} \epsilon$.

Theorem 5.2 For sufficiently small $\epsilon>0, Y^{\epsilon}(t) \rightarrow G$ as $t \uparrow \infty$.

Proof. By above theorem and the converse Liapunov theorem (Wilson (1969) [33, Theorem 3.2]), there exists a continuously differentiable Liapunov function for (17) that decreases monotonically along its trajectories outside $G$. Now use Theorem 1 of Hirsch (1989) [23, p. 339] to conclude that given any $\delta>0$, there exists an $\epsilon_{0}>0$ small enough such that $Y^{\epsilon}(t) \rightarrow$ the $\delta$-neighborhood of $G$. But once in a small neighborhood of $G, Y_{i_{0}}^{\epsilon}(t) \approx \sqrt{\pi\left(i_{0}\right)} \gamma^{*}>0$. Hence, if $\epsilon^{\prime}<\sqrt{\pi\left(i_{0}\right)} \gamma^{*}, 21$ reduces to (17) and the claim follows from the foregoing.

COROLlaRY $5.2 \phi^{T}\left(i_{0}\right) r^{\epsilon}(t) \rightarrow \gamma^{*}$ for sufficiently small $\epsilon>0$.

The last step left is to ensure 140:

Lemma $5.5 \sup _{n}\left\|r_{n}\right\|<\infty$, a.s.

Proof. Writing 15 as $\dot{r}(t)=h(r(t))$ for a suitably defined $h(\cdot)$, it is seen that $\lim _{a \uparrow \infty} \frac{h(a r)}{a}=-r$. Thus the 'scaling limit' of (15) as in Borkar and Meyn, (2000) is the o.d.e. $\dot{r}(t)=-r(t)$, which has the origin as its unique globally asymptotically stable limit point. The details are given in Theorem A.2 of the Appendix.

Our main result is now immediate:

THEOREM $5.3 \phi^{T}\left(i_{0}\right) r_{n} \rightarrow \gamma^{*}$ a.s.

Proof. Under (14), it is known that $r_{n} \rightarrow$ an internally chain transitive invariant set $L$ of (15) (see Benaim (1999) [6] and also Theorem A.1 of the Appendix for definitions and details). From Corollary $5.2, \phi^{T}\left(i_{0}\right) r=\gamma^{*} \forall r \in L$. The claim follows. 
6. The infinite dimensional case In this section we extend the above analysis to the case where the state space is a locally compact topological space. It may be noted that this is the case in many applications (e.g., finance). In order that similar arguments as before work in this set-up, we make certain additional assumptions which are satisfied in applications.

REMARK 6.1 Here we do not address the highly technical issue of well-posedness of the multiplicative Poisson equation on general state spaces. This has been extensively studied, e.g., in Kontoyiannis and Meyn (2003) [25] and Kontoyiannis and Meyn (2005) [26]. See also the works of Di Masi and Stettner [20], [21] for risk-sensitive control on general state spaces, which also address this issue.

The original problem is as in Section 2 and the fomulation of the algorithm is as in Section 4. Thus we do not repeat them here. This section is concerned with the convergence analysis when the state space is infinite.

Consider a locally compact topological space $S$ and a stationary Markov chain $\left\{X_{t}\right\}_{t \geq 0}$ on it. Let $P$ be the transition probability distribution for the Markov chain. Let $\nu$ be a regular Borel measure $S$. We assume that for each $x$ in $S$, the transition kernel $P(d y \mid x)$ is absolutely continuous and the density is strictly positive. Thus the Markov chain is irreducible. Let $\pi$ be a stationary distribution. Under the above assumption, the stationary distribution $\pi$ is absolutely continuous with respect to $\nu$ with strictly positive density, $\rho$, say. If $E$ denotes the real Hilbert space of real valued square integrable functions with respect to the measure $\pi$ on $S$, then $E$ can be given a partial order by declaring $f \geq g$ if $f(x) \geq g(x)$, $\pi$-almost everywhere on $S$. This partial order makes $E$ a Banach lattice with obvious supremum and infimum elements.

Given an $M$ dimensional feature vector $\phi(x)=\left(\phi_{1}(x), \ldots, \phi_{M}(x)\right)$ where $x \in S$, consider the subspace $X_{\phi}$ of $E$ defined as

$$
X_{\phi}=\left\{g \in E: g(x)=\sqrt{\rho(x)} \sum_{i=1}^{M} \phi_{i}(x) r_{i} \text { where } r_{1}, \ldots, r_{M} \text { are real numbers. }\right\}
$$

If $K_{1}, \ldots, K_{M}$ are non-empty disjoint closed subsets of $S$, then let $c_{i}=\int_{K_{i}} \rho d \pi$ and choose $\phi_{i}$ to be $\left(c_{i}\right)^{-1 / 2}$ times the indicator of $K_{i}$ for $i=1, \ldots, M$. From now on, we shall take the $\phi_{i}$ to be of this form. With this fixed choice of $\phi$, we shall denote $X_{\phi}$ simply by $X$. If $E_{+}$(respectively $E_{-}$) denotes the positive (respectively negative) cone in $E$, then the $\phi_{i}$ defined above are orthogonal functions in $E_{+}$. A short computation then shows that $\sum_{j=1}^{M} \int_{K_{j} \times K_{j}} \phi_{i}(x) \phi_{k}(y) d \pi(x) d \pi(y)=0$ for $i \neq k$. Thus if $\Phi$ is the $M \times M$ matrix valued function on $S$ whose $(i, j)$ th entry is $\phi_{i}(z) \phi_{j}(z)$, the matrix

$$
B \stackrel{\text { def }}{=} \int_{S} \Phi(z) \pi(d z)
$$

has orthogonal rows and hence is invertible. Clearly $B$ is a positive definite matrix. Consider the kernel

$$
k(x, y) \stackrel{\text { def }}{=} \sqrt{\rho(x)}\left\langle B^{-1 / 2} \phi(x), B^{-1 / 2} \phi(y)\right\rangle_{\mathbb{R}^{M}} \sqrt{\rho(y)}
$$

and associate with it the operator

$$
\Pi f(x) \stackrel{\text { def }}{=} \int_{S} k(x, y) f(y) d \nu(y), x \in S .
$$

Now, for $x, y, z \in S$, we have

$$
\begin{array}{rl}
\int_{S} & k(z, x) k(x, y) d \nu(x) \\
& =\sqrt{\rho(z)} \int_{S}\left\langle\phi(z), B^{-1} \phi(x)\right\rangle_{\mathbb{R}^{M}} \rho(x)\left\langle\phi(x), B^{-1} \phi(y)\right\rangle_{\mathbb{R}^{M}} d \nu(x) \sqrt{\rho(y)} \\
& =\sqrt{\rho(z) \rho(y)} \int_{S}\left\langle\phi(z), B^{-1} \phi(x)\right\rangle_{\mathbb{R}^{M}}\left\langle B^{-1} \phi(y), \phi(x)\right\rangle_{\mathbb{R}^{M}} d \pi(x) \\
& =\sqrt{\rho(z) \rho(y)} \int_{S}\left\langle\phi(z) \otimes B^{-1} \phi(y), \phi(x) \otimes B^{-1} \phi(x)\right\rangle_{\mathbb{R}^{M} \otimes \mathbb{R}^{M}} d \pi(x)
\end{array}
$$




$$
\begin{aligned}
& =\sqrt{\rho(z) \rho(y)}\left\langle\phi(z) \otimes B^{-1} \phi(y), \int_{S}\left(\phi(x) \otimes B^{-1} \phi(x)\right) d \pi(x)\right\rangle_{\mathbb{R}^{M} \otimes \mathbb{R}^{M}} \\
& =\sqrt{\rho(z) \rho(y)}\left\langle\left(I \otimes B^{-1}\right)(\phi(z) \otimes \phi(y)),\left(I \otimes B^{-1}\right) \int_{S}(\phi(x) \otimes \phi(x)) d \pi(x)\right\rangle_{\mathbb{R}^{M} \otimes \mathbb{R}^{M}} \\
& =\sqrt{\rho(z) \rho(y)}\left\langle\left(I \otimes B^{-1}\right)(\phi(z) \otimes \phi(y)),\left(I \otimes B^{-1}\right) \sum_{i=1}^{M} \pi\left(K_{i}\right)\left(e_{i} \otimes e_{i}\right)\right\rangle_{\mathbb{R}^{M} \otimes \mathbb{R}^{M}} \\
& =\sqrt{\rho(z) \rho(y)} \sum_{i=1}^{M} \pi\left(K_{i}\right)\left\langle\left(I \otimes\left(B B^{t}\right)^{-1}\right)(\phi(z) \otimes \phi(y)), e_{i} \otimes e_{i}\right\rangle_{\mathbb{R}^{M} \otimes \mathbb{R}^{M}} \\
& =\sqrt{\rho(z) \rho(y)} \sum_{i=1}^{M}\left(\pi\left(K_{i}\right)\right)^{-1} \phi_{i}(z) \phi_{i}(y) \\
& =\sqrt{\rho(z)}\left\langle\phi(z), B^{-1} \phi(y)\right\rangle_{\mathbb{R}^{M}} \sqrt{\rho(y)}=k(z, y) .
\end{aligned}
$$

This shows that the operator $\Pi$ is an idempotent. An elementary computation shows that it is symmetric too. So it is a projection. Since the map $f \rightarrow \int_{S} \phi(y) f(y) d \pi(y)$ is a surjective linear operator from $E$ onto $\mathbb{R}^{M}$, the closed subspace that corresponds to the projection $\Pi$ is the subspace $X$ defined above. Thus $\Pi$ is a finite-dimensional projection. By a positive operator on $E$ we shall mean a linear operator which takes non-negative functions to non-negative functions, unless specifically mentioned otherwise in the context. It is easy to check that $\Pi$ is a positive operator.

Given a running cost function $c: S \rightarrow \mathbb{R}$ which is assumed to be bounded on $S$, associate with it the linear operator $\mathcal{M}: E \rightarrow E$ defined by

$$
\mathcal{M} f(x) \stackrel{\text { def }}{=} \int_{S} \sqrt{\frac{\rho(x)}{\rho(y)}} e^{c(x, y)} f(y) P(d y \mid x) .
$$

Clearly $\mathcal{M}$ is a positive operator. Let $Q=\Pi \mathcal{M}$.

LEMMA 6.1 The operator $Q$ is a positive finite rank operator with a positive spectral radius $\gamma^{*}$.

Proof. The positivity of $Q$ is clear because it is a composition of two positive operators.

To prove that $\mathrm{Q}$ has a positive spectral radius $\gamma^{*}$, we need only to show that there exists a nonzero eigenvalue $\lambda$ in its spectrum, i.e., there is a non-zero $f$ in $E$ such that $Q f=\lambda f$, for some $\lambda \neq 0$. We shall produce an $f$ from $X$ such that $\Pi \mathcal{M} f=\lambda f$ for some positive $\lambda$. For this to happen, $\mathcal{M} f$ must be equal to $\lambda f+g$ for some $g$ orthogonal to $X$, because $Q f$ is the projection of $\mathcal{M} f$ onto the subspace $X$. Any $f$ in $X$ is of the form $\sum_{i=1}^{M} r_{i} \sqrt{\rho} \phi_{i}$ and hence $(\mathcal{M}-\lambda I) f$ is of the form $\sum_{i=1}^{M} r_{i}(\mathcal{M}-\lambda I) \sqrt{\rho} \phi_{i}$. For this to be orthogonal to $X$, we need

$$
\left\langle\sum_{i=1}^{M} r_{i}(\mathcal{M}-\lambda I) \sqrt{\rho} \phi_{i}, \sum_{j=1}^{M} \beta_{j} \sqrt{\rho} \phi_{j}\right\rangle_{E}=0
$$

for all $\beta_{1}, \ldots, \beta_{M} \in \mathbb{R}$. Using the exact forms of $\phi_{i}$, now it is easy to see that the left hand side of the above is the same as

$$
\sum_{i=1}^{M} \sum_{j=1}^{M} r_{i} \beta_{j}\left\langle\mathcal{M} \sqrt{\rho} \phi_{i}, \sqrt{\rho} \phi_{j}\right\rangle_{E}-\lambda \sum_{i=1}^{M} r_{i} \beta_{i} .
$$

So now if we define $A$ to be the $M \times M$ matrix whose $(i, j)$-th entry is the non-negative number $\left\langle\mathcal{M} \sqrt{\rho} \phi_{i}, \sqrt{\rho} \phi_{j}\right\rangle_{E}$, then $(22)$ above is equivalent to $\langle(A-\lambda I) \beta, r\rangle_{\mathbb{R}^{M}}=0$. Thus finding an $f$ in $X$ and a $\lambda>0$ to suit our purpose reduces to finding an $r \in \mathbb{R}^{M}$ and a $\lambda>0$ such that $r$ is orthogonal to $(A-\lambda I) \beta$. But the matrix $A$ has a Perron-Frobenius eigenvalue $\lambda>0$ (see for example Bapat and Raghavan (1997) 4, Theorem 1.4.4(i)]). Since $A-\lambda I$ is not of full-rank, we get a non-zero $r$ in $\mathbb{R}^{M}$ which is orthogonal to Range $(A-\lambda I)$.

COROLlary 6.1 The operator $Q$ has a positive eigenfunction $f^{*} \in X$ with $\gamma^{*}>0$ as the corresponding eigenvalue. 
Proof. This is a straightforward application of Krein-Rutman theorem (Theorem 7.10, page 277 of (Abramovich and Aliprantis, (2002)).

Let $H$ as before be $\left\{Y \in X: Q Y=\gamma^{*} Y, Y \geq 0, Y\left(i_{0}\right)>0\right\}$ for a fixed $i_{0} \in S$. Consider the o.d.e.

$$
\dot{Y}(t)=\left(\frac{Q}{\gamma^{*}}-I\right) Y(t) \text {. }
$$

Lemma 6.2 Every solution of (23) converges to $H$.

Proof. We omit the proof because it is similar to that of Lemma 2, now using the irreducibilty of the Markov chain.

Using the formula for the $\phi_{i}$, it is clear that the representation of an element $f$ of $X$ as $\sqrt{\rho(\cdot)} \phi^{T}(\cdot) r$ is unique. Thus one can define an invertible linear transformation $L$ between the two finite dimensional spaces $X$ and $\mathbb{R}^{M}$ as $L f \stackrel{\text { def }}{=} r$ where $f$ is as above. Associate the following $M \times M$ matrix $A$ with the cost function:

$$
A \stackrel{\text { def }}{=} \int_{S} \phi(x)\left(\int_{S} e^{c(x, y)} P(d y \mid x) \phi^{T}(y)\right) d \pi(x) .
$$

Using the fact that every $f \in X$ is of the form

$$
f(x)=\sqrt{\rho(x)}\langle\phi(x), r\rangle_{\mathbb{R}^{M}},
$$

a straightforward verification yields

$$
L \Pi \mathcal{M}=B^{-1} A L
$$

on elements of $X$. Consider now the ODE

$$
\dot{Y}_{t}=\frac{Q Y_{t}}{\phi^{T}\left(i_{0}\right) L Y_{t}}-Y_{t}=\left(\frac{\sqrt{\rho\left(i_{0}\right)} Q}{Y_{t}\left(i_{0}\right)}-I\right) Y_{t} .
$$

where $Y_{t} \in X$ and $i_{0}$ is a fixed state in $S$. By the help of the relation above it is easy to see that $Y_{t}(x)=\sqrt{\rho(x)}\langle\phi(x), r(t)\rangle_{\mathbb{R}^{M}}$ in $X$ satisfies the equation 24 if and only if $r(t)$ satisfies

$$
\dot{r}(t)=\left(\frac{B^{-1} A}{\phi^{T}\left(i_{0}\right) r(t)}-I\right) r(t) .
$$

Define $\mathcal{P}$ to be the positive cone in $X$. Thus the eigenvector $f^{*}$ obtained in Corollary 6.1 is in $\mathcal{P}$. It is straightforward to see that under condition 200, the conclusions of Lemma 5.3, Lemma 5.4 and Corollary 5.1 continue to be valid in this case, while the proofs require suitable modifications. In particular, the unique proper invariant set is $\left\{\sqrt{\rho\left(i_{0}\right)} \gamma^{*} Y^{*} / Y_{i_{0}}^{*}\right\}$. Now define

$$
G=\left\{f \in H: f\left(i_{0}\right)=\sqrt{\rho\left(i_{0}\right)} \gamma^{*}\right\} .
$$

Clearly $G$ is closed and bounded and hence compact in $X$. So it is compact in $E$. It is also convex and finally non-empty. An argument exactly similar to the proof of Theorem 5.1 with $\pi$ replaced by the density $\rho$ shows that $G$ is the globally exponentially stable attractor for 24 . Finally, we consider the equation of interest, viz.,

$$
\dot{r}(t)=\left(\frac{B^{-1} A}{\phi^{T}\left(i_{0}\right) r(t) \vee \epsilon}-I\right) r(t) .
$$

The bijection $L$ allows us to show that $r^{\epsilon}(t)$ satisfies $(26)$ if and only if

$$
Y^{\epsilon}(t)(x)=\sqrt{\rho(x)}\left\langle\phi(x), r^{\epsilon}(t)\right\rangle_{\mathbb{R}^{M}}
$$

in $X$ satisfies the equation

$$
\dot{Y}^{\epsilon}(t)=\left(\frac{\sqrt{\rho\left(i_{0}\right)} \Pi \mathcal{M}}{Y_{i_{0}}^{\epsilon}(t) \vee \epsilon^{\prime}}-I\right) Y^{\epsilon}(t)
$$

for $\epsilon^{\prime} \stackrel{\text { def }}{=} \sqrt{\rho\left(i_{0}\right)} \epsilon$. The rest of the analysis follows a route similar to Theorem 5.2 and we finally get an analogue of Corollary 5.2:

THEOREM $6.1 \phi^{T}\left(i_{0}\right) r^{\epsilon}(t) \rightarrow \gamma^{*}$ for sufficiently small $\epsilon>0$. 
By exactly the same arguments as for the finite dimensional case, we then have:

COROllary 6.2 The iterates $\left\{r_{n}\right\}$ satisfy: $\phi^{T}\left(i_{0}\right) r_{n} \rightarrow \gamma^{*}$ a.s.

It may be noted that the foregoing depends on our choice of $K_{1}, \ldots, K_{M}$. How to make a 'good' choice thereof is an issue that needs further study.

7. Conclusion The main contribution of this article has been to provide a rigorously justified linear function approximation based learning scheme for policy evaluation in risk-sensitive control. Some of the open issues that remain are:

(i) Better bounds on approximation error.

(ii) A recipe for choosing good basis functions.

(iii) Extension to unbounded 'running cost' $c(\cdot, \cdot)$ in the continuous state space case.

(iv) A rigorously justified scheme for learning optimal policy, with accompanying error bounds.

Appendix A. Here we outline the essential proofs in the 'o.d.e.' approach to the analysis of our algorithm, that have been used in the main text of the article. To begin with, we assume

$$
\sup _{n}\left\|r_{n}\right\|<\infty \text { a.s., }
$$

a fact we prove later in Theorem A.2 below. Define $h: \mathcal{R}^{M} \rightarrow \mathcal{R}^{M}$ by

$$
h(x) \stackrel{\text { def }}{=}\left(\frac{B^{-1} A}{\phi^{T}\left(i_{0}\right) x \vee \epsilon}-I\right) x .
$$

Note that $h(\cdot)$ is Lipschitz, i.e., $\|h(x)-h(y)\| \leq L\|x-y\|$ for some $0<L<\infty$. The equation (9) may be now rewritten as:

$$
r_{n+1}=r_{n}+a(n)\left(h\left(r_{n}\right)+Z_{n} r_{n}\right), n \geq 0,
$$

where $Z_{n} \rightarrow 0$, a.s. The limiting o.d.e. which $(30)$ might be expected to track asymptotically is

$$
\dot{r}(t)=h(r(t)), t \geq 0 \text {. }
$$

Let us construct a suitable continuous interpolated trajectory $\bar{r}(t), t \geq 0$ as follows:

Define time instants $t(0)=0, t(n)=\sum_{m=0}^{n-1} a(m), n \geq 1$. By our conditions on $\{a(n)\}, t(n) \uparrow \infty$. Let $I_{n} \stackrel{\text { def }}{=}[t(n), t(n+1)], n \geq 0$. Let

$$
\bar{r}(t)=r_{n}+\left(r_{n+1}-r_{n}\right) \frac{t-t(n)}{t(n+1)-t(n)}, t \in I_{n}
$$

where $\bar{r}(t(n))=r_{n}, n \geq 0$. Note that $\sup _{t \geq 0}\|\bar{r}(t)\|=\sup _{n}\left\|r_{n}\right\|<\infty$ a.s. Let $r^{s}(t), t \geq s$, denote the unique solution to 31 'starting at s':

$$
\dot{r}^{s}(t)=h\left(r^{s}(t)\right), t \geq s
$$

with $r^{s}(s)=\bar{r}(s), s \in \mathcal{R}$. Likewise, let $r_{s}(t), t \leq s$, denote the unique solution to (31) 'ending at s':

$$
\dot{r}_{s}(t)=h\left(r_{s}(t)\right), t \leq s
$$

with $r_{s}(s)=\bar{r}(s), s \in \mathcal{R}$. Define also

$$
\zeta_{n}=\sum_{m=0}^{n-1} a(m) Z_{m} r_{m}, n \geq 1 .
$$

Lemma A.1 For any $T>0$,

(i) $\lim _{s \rightarrow \infty} \sup _{t \in[s, s+T]}\left\|\bar{r}(t)-r^{s}(t)\right\|=0$, a.s.

(ii) $\lim _{s \rightarrow \infty} \sup _{t \in[s-T, s]}\left\|\bar{r}(t)-r_{s}(t)\right\|=0$, a.s. 
Proof We shall only prove the first claim as the second one can be proved analogously. Let $t(n+m)$ be in $[t(n), t(n)+T]$ and define $\hat{m}(n)=\min \{m>n: t(m) \geq t(n)+T\}$. Then $0<t(\hat{m}(n))-t(n) \leq T+K_{0}$, where $K_{0}$ is any bound on $\sup _{n} a(n)$. Let $[t]=\max \{t(k): t(k) \leq t\}$. Then by construction,

$$
\bar{r}(t(n+m))=\bar{r}(t(n))+\sum_{k=0}^{m-1} a(n+k) h(\bar{r}(t(n+k)))+\delta_{n, n+m},
$$

where $\delta_{n, n+m} \stackrel{\text { def }}{=} \zeta_{n+m}-\zeta_{n}$. Compare this with

$$
\begin{aligned}
r^{t(n)}(t(n+m)) & =\bar{r}(t(n))+\int_{t(n)}^{t(n+m)} h\left(r^{t(n)}(t)\right) d t \\
& =\bar{r}(t(n))+\sum_{k=0}^{m-1} a(n+k) h\left(r^{t(n)}(t(n+k))\right) \\
& +\int_{t(n)}^{t(n+m)}\left(h\left(r^{t(n)}(t)\right)-h\left(r^{t(n)}([t])\right)\right) d t .
\end{aligned}
$$

We shall first bound the integral on the r.h.s. Let $C_{0} \stackrel{\text { def }}{=} \sup _{n}\left\|r_{n}\right\|<\infty$ a.s., and let $t \in[s, s+T]$. Since $r^{s}(t)=\bar{r}(s)+\int_{s}^{t} h\left(r^{s}(u)\right) d u$ and $\left\|h\left(r^{s}(t)\right)\right\| \leq\|h(0)\|+L\left\|r^{s}(t)\right\|$, we have

$$
\left\|r^{s}(t)\right\| \leq\left(C_{0}+\|h(0)\| T\right)+L \int_{s}^{t}\left\|r^{s}(u)\right\| d u .
$$

By Gronwall's inequality, (38) implies

$$
\left\|r^{s}(t)\right\| \leq\left(C_{0}+\|h(0)\| T\right) e^{L T}, t \in[s, s+T]
$$

and thus for all $t \in[s, s+T]$, we have

$$
\left.\left\|h\left(r^{s}(t)\right)\right\| \leq C_{T} \stackrel{\text { def }}{=}\|h(0)\|+L\left(C_{0}+\|h(0)\| T\right) e^{L T}\right)<\infty \text { a.s. }
$$

Now, if $0 \leq k \leq(m-1)$, and $t \in(t(n+k), t(n+k+1)]$

$$
\left\|r^{t(n)}(t)-r^{t(n)}(t(n+k))\right\| \leq\left\|\int_{t(n+k)}^{t} h\left(r^{t(n)}(u)\right) d u\right\| \leq C_{T} a(n+k) .
$$

Thus,

$$
\begin{aligned}
& \left\|\int_{t(n)}^{t(n+m)}\left(h\left(r^{t(n)}(t)\right)-h\left(r^{t(n)}([t])\right)\right) d t\right\| \\
& \quad \leq L \sum_{k=0}^{m-1} \int_{t(n+k)}^{t(n+k+1)}\left\|r^{t(n)}(t)-r^{t(n)}(t(n+k))\right\| d t \\
& \quad \leq C_{T} L \sum_{k=1}^{\infty} a(n+k)^{2} \stackrel{n \uparrow \infty}{\longrightarrow} 0 \text { a.s. }
\end{aligned}
$$

Also, defining $\hat{\delta}_{n}=\sup _{1 \leq k \leq \hat{m}(n)-n}\left\|\delta_{n, n+k}\right\|$, since $Z_{n} \rightarrow 0$ a.s., we have

$$
\hat{\delta}_{n} \leq\left(T+K_{0}\right) C_{0} \sup _{k \geq n}\left\|Z_{k}\right\| \stackrel{n \uparrow \infty}{\longrightarrow} 0 \text { a.s. }
$$

Subtracting (37) from (36) and taking norms, we have,

$$
\begin{aligned}
& \left\|\bar{r}(t(n+m))-r^{t(n)}(t(n+m))\right\| \\
& \quad \leq L \sum_{k=0}^{m-1} a(n+k)\left\|\bar{r}(t(n+k))-r^{t(n)}(t(n+k))\right\| \\
& \quad+C_{T} L \sum_{k \geq 0} a(n+k)^{2}+\hat{\delta}_{n}
\end{aligned}
$$


a.s. Define $K_{T, n}=C_{T} L \sum_{k \geq 0} a(n+k)^{2}+\hat{\delta}_{n}$. Then $K_{T, n} \rightarrow 0$ a.s. as $n \rightarrow \infty$. Also, let $z_{k}=$ $\left\|\bar{r}(t(n+k))-r^{t(n)}(t(n+k))\right\|$, and $b_{k} \stackrel{\text { def }}{=} a(n+k)$. Then the above inequality 44 becomes:

$$
z_{m} \leq K_{T, n}+L \sum_{k=0}^{m-1} b_{k} z_{k}
$$

Note that $z_{0}=0$ and $\sum_{k=0}^{m-1} b_{k} \leq T$. From the discrete Gronwall lemma, we have

$$
\sup _{0 \leq k \leq m} z_{k} \leq K_{T, n} e^{L T}
$$

Then one has that for $t(n+m) \leq t(n)+T$,

$$
\left\|\bar{r}(t(n+m))-r^{t(n)}(t(n+m))\right\| \leq K_{T, n} e^{L T} \text { a.s. }
$$

If $t \in[t(n+k), t(n+k+1)]$, we have that $\bar{r}(t)=\lambda \bar{r}(t(n+k))+(1-\lambda) \bar{r}(t(n+k+1))$ for some $\lambda \in[0,1]$. Thus,

$$
\begin{aligned}
& \left\|r^{t(n)}(t)-\bar{r}(t)\right\| \\
& \quad=\left\|\lambda\left(r^{t(n)}(t)-\bar{r}(t(n+k))\right)+(1-\lambda)\left(r^{t(n)}(t)-\bar{r}(t(n+k+1))\right)\right\| \\
& \quad \leq \lambda\left\|r^{t(n)}(t(n+k))-\bar{r}(t(n+k))+\int_{t(n+k)}^{t} h\left(r^{t(n)}(u)\right) d u\right\| \\
& \quad+(1-\lambda)\left\|r^{t(n)}(t(n+k+1))-\bar{r}(t(n+k+1))-\int_{t}^{t(n+k+1)} h\left(r^{t(n)}(u)\right) d u\right\| \\
& \quad \leq(1-\lambda)\left\|r^{t(n)}(t(n+k+1))-\bar{r}(t(n+k+1))\right\| \\
& \quad+\lambda\left\|r^{t(n)}(t(n+k))-\bar{r}(t(n+k))\right\| \\
& \quad+\max (\lambda, 1-\lambda) \int_{t(n+k)}^{t(n+k+1)}\left\|h\left(r^{t(n)}(u)\right)\right\| d u .
\end{aligned}
$$

Since $\left\|h\left(r^{s}(t)\right)\right\| \leq C_{T}$ for all $t \in[s, s+T]$, it follows that

$$
\sup _{t \in[t(n), t(n)+T]}\left\|\bar{r}(t)-r^{t(n)}(t)\right\| \leq K_{T, n} e^{L T}+C_{T} a(n+k) \text { a.s. }
$$

The claim now follows for the special case of $s \rightarrow \infty$ along $\{t(n)\}$. The general claim follows easily from this.

A closed set $A \subset \mathcal{R}^{M}$ is said to be an invariant set for the o.d.e. 31] if any trajectory $r(t)$, $-\infty<t<\infty$, of (31) with $r(0) \in A$ satisfies $r(t) \in A \forall t \in \mathcal{R}$. It is said to be internally chain transitive in addition if for any $r, r^{\prime} \in A$ and any $\epsilon>0, T>0$, there exists $n=n(\epsilon, T) \geq 1$ and points $r_{0}=r, r_{1}, \ldots, r_{n-1}, r_{n}=r^{\prime}$ in $A$ such that the trajectory of (31) initiated at $r_{k}$ meets the $\epsilon$-neighbourhood of $r_{k+1}$ for $0 \leq k<n$ after a time $\geq T$. (If we restrict to $r=r^{\prime}$ in the above, the set is said to be internally chain recurrent.) Let $\Phi_{t}: \mathcal{R}^{M} \rightarrow \mathcal{R}^{M}$ denote the map that maps $r(0)$ to $r(t)$ via (31). Under our definition (29) of $h(\cdot)$, this map will be continuous (in fact Lipschitz) for each $t>0$.

Our general convergence theorem for stochastic approximation is the following:

Theorem A.1 Almost surely, the sequence $\left\{r_{n}\right\}$ generated by (30) converges to a (possibly sample path dependent) compact connected internally chain transitive invariant set of (31).

Proof Consider a sample point where $(28)$ and the conclusions of Lemma A.1 hold. Let $A$ denote the set $\cap_{t \geq 0} \overline{\{\bar{r}(s): s \geq t\}}$. Since $\bar{r}(\cdot)$ is continuous and bounded, $\overline{\{\bar{r}(s): s \geq t\}}, t \geq 0$, is a nested family of nonempty compact and connected sets. A, being the intersection thereof, will also be nonempty, compact and connected. Then $\bar{r}(t) \rightarrow A$ and therefore $r_{n} \rightarrow A$. In fact, for any $\epsilon>0$, let $A \stackrel{\text { def }}{=}\{r$ : $\left.\min _{r^{\prime} \in A}\left\|r-r^{\prime}\right\|<\epsilon\right\}$. Then $\left(A^{\epsilon}\right)^{c} \cap A=\phi$. Hence by the finite intersection property of families of compact sets, $\left(A^{\epsilon}\right)^{c} \cap \overline{\left\{\bar{r}(s): s \geq t^{\prime}\right\}}=\phi$ for some $t^{\prime}>0$. That is, $\bar{r}\left(t^{\prime}+\cdot\right) \in A^{\epsilon}$. Conversely, if $r \in A$, there exists $s_{n} \uparrow \infty$ in $[0, \infty)$ such that $\bar{r}\left(s_{n}\right) \rightarrow r$. This is immediate from the definition of $A$. In fact, we have

$$
\max _{s \in[t(n), t(n+1)]}\|\bar{r}(s)-\bar{r}(t(n))\|=O(a(n)) \rightarrow 0
$$


as $n \rightarrow \infty$. Thus we may take $s_{n}=t(m(n))$ for suitable $\{m(n)\}$ without any loss of generality. Let $\tilde{r}(\cdot)$ denote the trajectory of (31) with $\tilde{r}(0)=r$. Then, by Lemma A.1(1) and the continuity of the map $\Phi_{t}$ defined above, it follows that $r^{s_{n}}\left(s_{n}+t\right)=\Phi_{t}\left(\bar{r}\left(s_{n}\right)\right) \rightarrow \Phi_{t}(r)=\tilde{r}(t)$ for all $t>0$. By Lemma A.1, $\bar{r}\left(s_{n}+t\right) \rightarrow \tilde{r}(t)$, implying that $\tilde{r}(t) \in A$ as well. A similar argument works for $t<0$, using Lemma A.1(2). Thus $A$ is invariant under (31).

Let $\tilde{r}_{1}, \tilde{r}_{2} \in A$ and fix $\epsilon>0, T>0$. Pick $\frac{\epsilon}{4}>\delta>0$ such that: if $\left\|r-r^{\prime}\right\|<\delta$ and $\hat{r}_{r}(\cdot), \hat{r}_{r^{\prime}}(\cdot)$ are solutions to (31) with initial conditions $r, r^{\prime}$ resp., then $\max _{t \in[0,2 T]}\left\|\hat{r}_{r}(t)-\hat{r}_{r^{\prime}}(t)\right\|<\frac{\epsilon}{4}$. Also pick $n_{0}>1$ such that $s \geq t\left(n_{0}\right)$ implies that $\bar{r}(s+\cdot) \in A^{\delta}$ and $\sup _{t \in[s, s+2 T]}\left\|\bar{r}(t)-r^{s}(t)\right\|<\delta$. Pick $n_{2}>n_{1} \geq n_{0}$ such that $\left\|\bar{r}\left(t\left(n_{k}\right)\right)-\tilde{r}_{k}\right\|<\delta, k=1,2$. Let $k T \leq t\left(n_{2}\right)-t\left(n_{1}\right)<(k+1) T$ for some integer $k \geq 0$ and let $s(0)=t\left(n_{1}\right), s(j)=s(0)+j T, 1 \leq j<k, s(k)=t\left(n_{2}\right)$. Then for $0 \leq j<k, \sup _{t \in[s(j), s(j+1)]}\left\|\bar{r}(t)-r^{s(j)}(t)\right\|<\delta$. Pick $\hat{r}_{j}, 0 \leq j \leq k$, in $A$ such that $\hat{r}_{1}=\tilde{r}_{1}, \hat{r}_{k}=\tilde{r}_{2}$, and for $0<j<k, \hat{r}_{j}$ 's are in the $\delta$-neighbourhood of $\bar{r}(s(j))$. The sequence $\left(s(j), \hat{r}_{j}\right), 0 \leq j \leq k$, verifies the definition of internal chain transitivity: If $r_{j}^{\star}(\cdot)$ denote the trajectories of 31 initiated at $\hat{r}_{j}$ for each $j$, we have

$$
\begin{aligned}
& \left\|r_{j}^{\star}(s(j+1)-s(j))-\hat{r}_{j+1}\right\| \\
& \quad \leq\left\|r_{j}^{\star}(s(j+1)-s(j))-r^{s(j)}(s(j+1))\right\| \\
& \quad+\left\|r^{s(j)}(s(j+1))-\bar{r}(s(j+1))\right\|+\left\|\bar{r}(s(j+1))-\hat{r}_{j+1}\right\| \\
& \quad \leq \frac{\epsilon}{4}+\frac{\epsilon}{4}+\frac{\epsilon}{4}<\epsilon .
\end{aligned}
$$

This completes the proof.

We return to the task of proving 28. The functions $h_{c}(x) \triangleq h(c x) / c, c \geq 1, x \in \mathcal{R}^{M}$, satisfy : $h_{c}(x) \rightarrow h_{\infty}(x) \equiv-x$ as $c \rightarrow \infty$, uniformly on compacts. Furthermore,

(i) $h_{c}, h_{\infty}$ will be Lipschitz with the same Lipschitz constant $L$ as $h$, implying in particular a common linear growth condition applicable to all and the well-posedness of the o.d.e.

$$
\dot{r}(t)=h_{c}(r(t)), 1 \leq c \leq \infty,
$$

and,

(ii) the o.d.e.

$$
\dot{r}(t)=h_{\infty}(r(t))=-r(t)
$$

has the origin as its unique globally asymptotically stable equilibrium.

Let $\phi_{\infty}(t, r)$ (resp., $\left.\phi_{c}(t, r)\right)$ denote the solution of the o.d.e. (53) (resp. (52) ) with initial condition $r$. The following lemma is obvious for our case.

Lemma A.2 There exists a $T>0$ such that for all initial conditions $r$ on the unit sphere, $\left\|\phi_{\infty}(t, r)\right\|<\frac{1}{8}$ for all $t>T$.

The following lemma shows that the solutions of the o.d.e.s $\sqrt{53}$ and $(52)$ are close for $c$ large enough.

Lemma A.3 Let $K \subset \mathcal{R}^{M}$ be compact, and let $[0, T]$ be a given time interval. Then for $t \in[0, T]$ and $r_{0} \in K$,

$$
\left\|\phi_{c}(t, r)-\phi_{\infty}\left(t, r_{0}\right)\right\| \leq\left[\left\|r-r_{0}\right\|+\epsilon(c) T\right] e^{L T},
$$

where $\epsilon(c)$ is independent of $r_{0} \in K$ and $\epsilon(c) \rightarrow 0$ as $c \rightarrow \infty$. In particular, if $r=r_{0}$, then

$$
\left\|\phi_{c}\left(t, r_{0}\right)-\phi_{\infty}\left(t, r_{0}\right)\right\| \leq \epsilon(c) T e^{L T} .
$$

Proof Note that

$$
\begin{aligned}
\phi_{c}(t, r) & =r+\int_{0}^{t} h_{c}\left(\phi_{c}(s, r)\right) d s, \text { and } \\
\phi_{\infty}\left(t, r_{0}\right) & =r_{0}+\int_{0}^{t} h_{\infty}\left(\phi_{\infty}\left(s, r_{0}\right)\right) d s .
\end{aligned}
$$

This gives:

$$
\left\|\phi_{c}(t, r)-\phi_{\infty}\left(t, r_{0}\right)\right\| \leq\left\|r-r_{0}\right\|+\int_{0}^{t}\left\|h_{c}\left(\phi_{c}(s, r)\right)-h_{\infty}\left(\phi_{\infty}\left(s, r_{0}\right)\right)\right\| d s .
$$


Now, using the facts that $\phi_{\infty}([0, T], K)$ is compact, $h_{c} \rightarrow h_{\infty}$ uniformly on compact sets, and the Lipschitz property of $h_{c}$, we get

$$
\begin{aligned}
\| h_{c}\left(\phi_{c}(s, r)\right) & -h_{\infty}\left(\phi_{\infty}\left(s, r_{0}\right)\right) \| \\
& \leq\left\|h_{c}\left(\phi_{c}(s, r)\right)-h_{c}\left(\phi_{\infty}\left(s, r_{0}\right)\right)\right\| \\
& +\left\|h_{c}\left(\phi_{\infty}\left(s, r_{0}\right)\right)-h_{\infty}\left(\phi_{\infty}\left(s, r_{0}\right)\right)\right\| \\
& \leq L\left\|\phi_{c}(s, r)-\phi_{\infty}\left(s, r_{0}\right)\right\|+\epsilon(c),
\end{aligned}
$$

where $\epsilon(c)$ is independent of $r_{0} \in K$ and $\rightarrow 0$ as $c \rightarrow \infty$. Thus for $t \leq T$, we get

$$
\left\|\phi_{c}(t, r)-\phi_{\infty}\left(t, r_{0}\right)\right\| \leq\left\|r-r_{0}\right\|+\epsilon(c) T+L \int_{0}^{t}\left\|\phi_{c}(s, r)-\phi_{\infty}\left(s, r_{0}\right)\right\| d s .
$$

The conclusion follows from Gronwall's inequality.

The previous two lemmas give us:

Corollary A.1 There exist $c_{0}>0$ and $T>0$ such that for all initial conditions $r$ on the unit sphere, $\left\|\phi_{c}(t, r)\right\|<\frac{1}{4}$ for $t \in[T, T+1]$ and $c>c_{0}$.

Proof. Choose $T$ satisfying Lemma A.2 and $c$ such that $\epsilon(c)(T+1) e^{L(T+1)}<\frac{1}{8}$. Now, use equation (54).

Let $T_{0}=0$ and $T_{n+1}=\min \left\{t(m): t(m) \geq T_{n}+T\right\}$ for $n \geq 0$. Then $T_{n+1} \in\left[T_{n}+T, T_{n}+T+K_{0}\right] \forall n$, $T_{n}=t(m(n))$ for suitable $m(n) \uparrow \infty$, and $T_{n} \uparrow \infty$. For sake of some notational simplicity, let $K_{0}=1$ without any loss of generality. Define the piecewise continuous trajectory $\hat{r}(t), t \geq 0$, by $: \hat{r}(t)=\bar{r}(t) / \rho(n)$ for $t \in\left[T_{n}, T_{n+1}\right]$, where $\rho(n) \triangleq\left\|\bar{r}\left(T_{n}\right)\right\| \vee 1, n \geq 0$. That is, we obtain $\hat{r}(\cdot)$ from $\bar{r}(\cdot)$ by observing the latter at times $\left\{T_{n}\right\}$ that are spaced approximately $T$ apart. In case the observed value falls outside the unit ball of $\mathcal{R}^{M}$, it is reset to a value on the unit sphere of $\mathcal{R}^{M}$ by normalization. Not surprisingly, this prevents any possible blow-up of the trajectory, as reflected in the following lemma. For later use, we also define $\hat{r}\left(T_{n+1}^{-}\right) \triangleq \bar{r}\left(T_{n+1}\right) / \rho(n)$. This is the same as $\hat{r}\left(T_{n+1}\right)$ if there is no jump at $T_{n+1}$, and equal to $\lim _{t \uparrow T_{n+1}} \hat{r}(t)$ if there is one. For $n \geq 0$, let $r^{n}(t), t \in\left[T_{n}, T_{n+1}\right]$ denote the trajectory of (52) with $c=\rho(n)$ and $r^{n}\left(T_{n}\right)=\hat{r}\left(T_{n}\right)$.

Lemma A.4 $\sup _{t}\|\hat{r}(t)\|<\infty$ a.s. and, $\lim _{n \rightarrow \infty} \sup _{t \in\left[T_{n}, T_{n+1}\right]}\left\|\hat{r}(t)-r^{n}(t)\right\|=0$, a.s.

Proof. For simplicity, we assume $L>1, a(n)<1 \forall n$. Note that for $m(n) \leq k<m(n+1)$,

$$
\hat{r}(t(k+1))=\hat{r}(t(k))+a(k)\left(h_{\rho(n)}(\hat{r}(t(k)))+\hat{M}_{k+1}\right) .
$$

where $\hat{M}_{k+1} \stackrel{\text { def }}{=} \frac{r_{k} Z_{k}}{\rho(n)}$. This yields, for $0<k \leq m(n+1)-m(n)$,

$$
\begin{gathered}
\hat{r}(t(m(n)+k))=\hat{r}(t(m(n)))+\sum_{i=0}^{k-1} a(m(n)+i) h_{\rho(n)}(\hat{r}(t(m(n)+i))) \\
+\left(\hat{\zeta}_{m(n)+k}-\hat{\zeta}_{m(n)}\right),
\end{gathered}
$$

where $\hat{\zeta}_{n} \stackrel{\text { def }}{=} \sum_{j=0}^{n-1} a(j) \hat{M}_{j+1}, n \geq 1$. We have

$$
\left\|h_{\rho(n)}(\hat{r}(t(m(n)+i)))\right\| \leq\|h(0)\|+L\|\hat{r}(t(m(n)+i))\| .
$$

Also,

$$
\begin{aligned}
\hat{\zeta}_{m(n)+k}-\hat{\zeta}_{m(n)} & =\sum_{j=m(n)}^{j=m(n)+k-1} a(j) \hat{M}_{j+1} \\
& =\sum_{j=0}^{k-1} a(m(n)+j) Z_{m(n)+j} \hat{r}(t(m(n)+j)) .
\end{aligned}
$$

Furthermore, $\sum_{0 \leq i<m(n+1)-m(n)} a(m(n)+i) \leq T+1$. Therefore we get,

$$
\begin{aligned}
& \|\hat{r}(t(m(n)+k))\| \\
& \quad \leq\|\hat{r}(t(m(n)))\|+\sum_{i=0}^{k-1} a(m(n)+i)\left(\|h(0)\|+\left(L+\left\|Z_{m(n)+i}\right\|\right)\|\hat{r}(t(m(n)+i))\|\right) \\
& \quad \leq \sum_{i=0}^{k-1} a(m(n)+i)\left(L+\left\|Z_{m(n)+i}\right\|\right)\|\hat{r}(t(m(n)+i))\|+\|h(0)\|(T+1)+1,
\end{aligned}
$$


where we use the fact $\|\hat{r}(t(m(n)))\| \leq 1$. We can now apply the discrete Gronwall inequality to obtain

$$
\|\hat{r}(t(m(n)+k))\| \leq(\|h(0)\|(T+1)+1) e^{L(T+1)} e^{\sum_{j=0}^{k-1} a(m(n)+j)\left\|Z_{m(n)+j}\right\|}
$$

for $0<k \leq m(n+1)-m(n)$. Since $Z_{n} \stackrel{n \uparrow \infty}{\longrightarrow} 0$ a.s., we have

$$
\sum_{j=0}^{k-1} a(m(n)+j)\left\|Z_{m(n)+j}\right\| \leq(T+1) \sup _{n}\left\|Z_{n}\right\| \leq(T+1) K .
$$

for some bound $K>0$ independent of $n$. Hence we have,

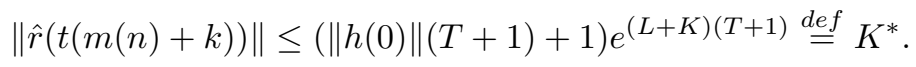

It follows that $\hat{r}(\cdot)$ remains bounded on $\left[T_{n}, T_{n+1}\right]$ by some (random) $K^{*}>0$ and this bound is independent of $n$. We can now mimic the argument of Lemma A.1 above to show that $\lim _{n \rightarrow \infty} \sup _{t \in\left[T_{n}, T_{n+1}\right]}\left\|\hat{r}(t)-r^{n}(t)\right\|=0$ a.s.

This leads to our main result:

Theorem A.2 Under above conditions, $\sup _{n}\left\|r_{n}\right\|<\infty$ a.s.

Proof. Fix a sample point where the claims of Lemma A.4 hold. We will first show that $\sup _{n}\left\|\bar{r}\left(T_{n}\right)\right\|<$ $\infty$. If this does not hold, there will exist a sequence $T_{n_{1}}, T_{n_{2}}, \ldots$ such that $\left\|\bar{r}\left(T_{n_{k}}\right)\right\| \nearrow \infty$, i.e., $\rho\left(n_{k}\right) \nearrow \infty$. We saw (Corollary A.1) that there exists a scaling factor $c_{0}>0$ and a $T>0$ such that for all initial conditions $r$ on the unit sphere, $\left\|\phi_{c}(t, r)\right\|<\frac{1}{4}$ for $t \in[T, T+1]$ and $c>c_{0}(\geq 1$ by assumption). If $\rho(n)>c_{0},\left\|\hat{r}\left(T_{n}\right)\right\|=\left\|r^{n}\left(T_{n}\right)\right\|=1$, and $\left\|r^{n}\left(T_{n+1}\right)\right\|<\frac{1}{4}$. But then by Lemma A.4, $\left\|\hat{r}\left(T_{n+1}^{-}\right)\right\|<\frac{1}{2}$ if $n$ is large. Thus, for $\rho(n)>c_{0}$ and $n$ sufficiently large,

$$
\frac{\left\|\bar{r}\left(T_{n+1}\right)\right\|}{\left\|\bar{r}\left(T_{n}\right)\right\|}=\frac{\left\|\hat{r}\left(T_{n+1}^{-}\right)\right\|}{\left\|\hat{r}\left(T_{n}\right)\right\|}<\frac{1}{2} .
$$

We conclude that if $\left\|\bar{r}\left(T_{n}\right)\right\|>c_{0}, \bar{r}\left(T_{k}\right), k \geq n$ falls back to the ball of radius $c_{0}$ at an exponential rate. Thus if $\left\|\bar{r}\left(T_{n}\right)\right\|>c_{0},\left\|\bar{r}\left(T_{n-1}\right)\right\|$ is either even greater than $\left\|\bar{r}\left(T_{n}\right)\right\|$ or is inside the ball of radius $c_{0}$. Then there must be an instance prior to $n$ when $\bar{r}(\cdot)$ jumps from inside this ball to outside the ball of radius $0.9 \rho(n)$. Thus, corresponding to the sequence $\rho\left(n_{k}\right) \nearrow \infty$, we will have a sequence of jumps of $\bar{r}\left(T_{n}\right)$ from inside the ball of radius $c_{0}$ to points increasingly far away from the origin. But, by the discrete Gronwall argument analogous to the one used in Lemma A.4, it follows that there is a bound on the amount by which $\|\bar{r}(\cdot)\|$ can increase over an interval of length $T+1$ when it is inside the ball of radius $c_{0}$ at the beginning of the interval. This leads to a contradiction. Thus $\tilde{C} \stackrel{\text { def }}{=} \sup _{n}\left\|\bar{r}\left(T_{n}\right)\right\|<\infty$. This implies that $\sup _{n}\left\|r_{n}\right\| \leq \tilde{C} K^{*}<\infty$ for $K^{*}$ as in 61.

Acknowledgments. Research of the first named author is supported in part by an Infosys Fellowship. The second named author thanks the Department of Science and Technology, Govt. of India for the Raja Ramanna Fellowship and also special assistance from the Universities Grants Commission, Govt. of India. The third named author's research is supported in part by a J. C. Bose Fellowship of Department of Science and Technology, Govt. of India. This author thanks Prof. Sean Meyn for some useful discussions.

\section{References}

[1] Y. A. Abramovich and C. D. Aliprantis, An Invitation to Operator Theory, American Mathematical Society, Providence, RI, 2002.

[2] A. Bagchi and K. Sureshkumar, Dynamic asset management: risk sensitive criterion with positive factors constraints, Recent Developments in Mathematical Finance (J. Yong ed.), World Scientific, Hong Kong, 2002, $1-11$.

[3] S. Balaji and S. P. Meyn, Multiplicative ergodicity and large deviations for an irreducible Markov chain, Stochastic Processes and Their Appl. 90 (2000), 123-144.

[4] R. B. Bapat and T. E. S. Raghavan, Nonnegative Matrices and Applications. Cambridge University Press, Cambridge, UK, 1997.

[5] A. G. Barto, R. S. Sutton and C. Anderson, Neuron-like elements that can solve difficult learning control problems, IEEE Transactions on Systems, Man and Cybernetics, 13 (1983), 835-846. 
[6] M. Benaim, Dynamics of stochastic approximation algorithms, Le Séminaire de Probabilités (J. Azéma, M. Emery, M. Ledoux and M. Yor, eds.), Springer Lecture Notes in Mathematics No. 1709, Springer Verlag, Berlin-Heidelberg, 1999, 1-68.

[7] A. Benveniste, M. Metivier and P. Priouret, Adaptive Algorithms and Stochastic Approximations. Springer Verlag, Berlin - Heidelberg, 1991.

[8] D. P. Bertsekas, V. S. Borkar and A. Nedic, Improved temporal difference methods with linear function approximation, Handbook of Learning and Approximate Dynamic Programming (J. Si, A. G. Barto, W. B. Powell, D. Wunsch, eds.), IEEE Press, New York, 2004, 235-259.

[9] D. P. Bertsekas and A. Nedic, Least squares policy evaluation algorithms with linear function approximation, Discrete Event Dynamical Systems, 13, 2003, 79-110.

[10] D. P. Bertsekas and J. N. Tsitsiklis, Neurodynamic Programming, Athena Scientific, Belmont, MA, USA, 1996.

[11] R. Bhatia, Matrix Analysis. Springer Verlag, New York, 1997.

[12] T. R. Bielecki and S. R. Pliska, Risk sensitive dynamic asset management, Appl. Math. Optim., 39 (1999), 337-360.

[13] T. R. Bielecki and S. R. Pliska, Risk sensitive asset management with transaction costs, Finance and Stochastics, 4 (2000), 1-33.

[14] T. R. Bielecki and S. R. Pliska, Economic properties of the risk-sensitive criterion for portfolio management, Rev. Account. Finance., 2 (2003), 3-17.

[15] V. S. Borkar, A sensitivity formula for risk-sensitive cost and the actor-critic algorithm, Systems and Control Letters, 44 (2001), 339-346.

[16] V. S. Borkar, Q-learning for risk-sensitive control, Math. Op. Research, 27 (2002), 294-311.

[17] V.S. Borkar and S. P. Meyn, The o.d.e. method for convergence of stochastic approximation and reinforcement learning, SIAM J. Control and Optim., 38 (2000), 447-469.

[18] V. S. Borkar, and S. P. Meyn, Risk-sensitive optimal control for Markov decision processes with monotone costs, Math. Op. Research, 27 (2002), 192-209.

[19] D. P. Derevitskii and A. L. Fradkov, Two models for analyzing the dynamics of adaptation algorithms, Automation and Remote Control, 35 (1974), 59-67.

[20] G. B. Di Masi and L. Stettner, Infinite horizon risk sensitive control of discrete time Markov processes under minorization property, Preprint available at 'http://www.impan.gov.pl/User/stettner/69.pdf'.

[21] G. B. Di Masi and L. Stettner, On additive and multiplicative (controlled) Poisson equations, Preprint available at 'http://www.impan.gov.pl/User/stettner/72.pdf'.

[22] G. H. Golub and C. F. Van Loan, Matrix Computations, Johns Hopkins University Press, Baltimore, MD, USA, 1996.

[23] M. W. Hirsch, Convergent activation dynamics in continuous time networks Neural Networks, 2 (1989), 331-349.

[24] I. Karatzas and S. E. Shreve, Brownian Motion and Stochastic Calculus. Springer-Verlag, New York, USA, 1988.

[25] I. Kontoyiannis and S. P. Meyn, Spectral theory and limit theorems for geometrically ergodic Markov processes, Anal. Appl. Probab. 13 (2003), 304-362.

[26] I. Kontoyiannis and S. P. Meyn, Large deviations asymptotics and the spectral theory of multiplicatively regular Markov processes, Electronic J. Probability, 10 (2005), 61-123.

[27] H. J. Kushner and P. Dupuis, Numerical Methods for Stochastic Control Problems in Continuous Time, Second ed. Springer-Verlag, New York, USA, 2001.

[28] J. Si, A. G. Barto, W. B. Powell and D. Wunsch, eds., Handbook of Learning and Approximate Dynamic Programming. IEEE Press, New York, USA, 2004.

[29] G. W. Stewart, Matrix Algorithms, Volume II. SIAM, Philadelphia, USA, 2001.

[30] R. S. Sutton and A. G. Barto, Reinforcement Learning. MIT Press, Cambridge, MA, USA, 1998.

[31] J. N. Tsitsiklis and B. Van Roy, An analysis of temporal-difference learning with function approximation, IEEE Transactions on Automatic Control 42 (1997), 674-690.

[32] C. J. C. H. Watkins, Learning from delayed rewards, Ph.D. Thesis, University of Cambridge, UK, 1989.

[33] F. W. Wilson, Smoothing derivatives of functions and applications, Transactions of American Mathematical Society, 139 (1969), 413-428. 